\title{
Les langues de France et l'Europe
}

\section{Philippe Martel}

\section{OpenEdition}

Journals

Édition électronique

URL : http://journals.openedition.org/ries/4229

DOI : $10.4000 /$ ries.4229

ISSN : 2261-4265

\section{Éditeur}

Centre international d'études pédagogiques

\section{Édition imprimée}

Date de publication : 1 septembre 1994

Pagination : 63-74

ISSN : 1254-4590

\section{Référence électronique}

Philippe Martel, «Les langues de France et l'Europe », Revue internationale d'éducation de Sèvres [En ligne], 03 | 1994, mis en ligne le 17 avril 2015, consulté le 02 mai 2019. URL : http:// journals.openedition.org/ries/4229 ; DOI : 10.4000/ries.4229

Ce document a été généré automatiquement le 2 mai 2019.

(c) Tous droits réservés 


\title{
Les langues de France et l'Europe
}

\author{
Philippe Martel
}

1 Voilà un titre qui peut surprendre. Ce que nous appelons ici «langues de France »alsacien et francique de Lorraine, basque, breton, catalan, corse, flamand, occitan -, il est plutôt d'usage en France de l'appeler "patois" ou dialectes régionaux - langues régionales au mieux. Il est aussi admis, par le sens commun français moyen, que ces idiomes, d'ailleurs en voie de disparition rapide sous les coups de la modernité, ne sont plus que le mode d'expression des derniers vestiges de la vieille société rurale et qu'ils n'ont pas vocation à dépasser le cadre restreint des réduits villageois où on peut encore les employer. Qu'iraient-ils donc faire dans cette Europe en construction, marchant d'un pas assuré - si tout va bien ! - vers le $\mathrm{XXI}^{\mathrm{e}}$ siècle ? Et à quoi bon poser la question de leur place dans le futur paysage culturel et linguistique d'un continent où bien des langues d'État ne sont même pas sûres de tirer leur épingle du jeu? Alors que l'enjeu le plus évident à première vue, pour un Français, c'est le maintien de sa langue nationale face à l'envahissement de l'anglais? Or, la question se pose vraiment. Mieux: ce sont les instances européennes elles-mêmes qui en sont venues à la poser explicitement, à travers un certain nombre d'initiatives et de textes officiels. Avec, il est vrai, plus ou moins de succès.

2 Ce que nous allons tenter de voir ici, c'est l'état actuel du problème, à la fois dans sa dimension institutionnelle - quelle place réserve la construction européenne aux petites langues parlées sur les territoires des États membres - et dans sa dimension plus largement culturelle, voire politique : au fond, à quoi peuvent servir, dans la constitution d'une culture européenne de type nouveau, ces langues en apparence condamnées? Sans oublier une question : les États membres en général, et la France en particulier, sont-ils vraiment disposés à accepter cette prise en compte des langues minoritaires à l'échelle de la Communauté?

\section{Langues de France, langues d'Europe}

3 Premier aspect, purement factuel: le problème des langues de France se pose d'autant plus facilement au niveau international que ces langues, précisément, loin d'être des 
spécificités purement françaises, participent plus largement du paysage linguistique européen dans son ensemble. C'est clair pour l'Alsace et la Lorraine germanophone, qui prolongent, de ce côté-ci du Rhin, le vaste espace linguistique allemand. Que les dialectes alsaciens et lorrains divergent par rapport au Hochdeutsch enseigné dans nos lycées, c'est l'évidence - et Dieu sait si la méfiance, historiquement quelque peu justifiée !, à l'égard du grand voisin oriental a pu amener des intellectuels alsaciens à exagérer la distance qui sépare leurs parlers de la langue officielle de l'Allemagne. Ceci dit, il n'existe pas un groupe dialectal alsacien que le Rhin séparerait opportunément du magma des parlers allemands ou un lorrain qui s'arrêterait prudemment aux poteaux frontières. Dans les deux cas, il n'y a pas de solution de continuité avec ce qui se parle de l'autre côté de la limite sacrée : traits alémaniques ou franciques se rient des tracés hérités des guerres passées, et on parle substantiellement la même chose à Strasbourg et à offenburg. Jusqu'en 1870 d'ailleurs, alors même que la francité de l'Alsace n'était mise en doute par personne, les imprimeries locales imprimaient sans état d'âme une respectable quantité de titres allemands - et pratiquement aucun en dialecte. Tandis qu'à l'heure actuelle, c'est l'allemand qui s'enseigne dans les régions germanophones de France, même si son acquisition peut se faire en partant du dialecte parlé.

4 L'allemand n'est pas la seule langue transfrontalière : le catalan et le basque sont dans la même situation, chevauchant, depuis toujours, la « frontière naturelle » des Pyrénées. Le flamand de France prolonge celui de Belgique - et, au-delà, non seulement l'aire néerlandophone mais aussi toute la zone dialectale qui couvre l'Allemagne du Nord. L'occitan est typologiquement très proche du catalan; par ailleurs, lui aussi franchit les frontières : on le parle dans la montagne piémontaise, côté italien, et en Val d'Aran " espagnol » - c'est même là le seul endroit où il jouisse d'une véritable reconnaissance officielle. Même le breton n'est pas séparable de l'ensemble celtique, tel qu'il subsiste dans les îles britanniques - et ce en dépit du fait que la distance qui le sépare de son plus proche parent, le gallois, est assez considérable. Quant au corse, c'est seulement depuis quelques années que l'évolution de la conscience linguistique (pas seulement linguistique) sur l'île a pu amener ses défenseurs à le constituer en langue autonome alors même que sa structure le rapproche sans doute possible des dialectes de l'Italie centrale et que la langue de culture de la Corse a longtemps été le toscan. Ici un facteur sociolinguistique - la volonté d'identification des locuteurs - l'emporte sur les réalités dialectologiques, sans pour autant casser définitivement le lien: l'auditeur ou le téléspectateur corse peut accéder sans problèmes aux émissions des médias italiens.

Bref: pratiquement toutes les langues parlées en France le sont aussi en dehors, et constituent des ponts entre la France et ses voisins. C'est bien pourquoi d'ailleurs on les a parfois tenues pour suspectes: ce que Barère attaquait en 1794, c'était moins les " patois » en général que les idiomes frontaliers, susceptibles de lier les populations qui les parlaient avec celles d'États hostiles à la République. Et ce n'est pas un hasard si la politique linguistique la plus ouvertement répressive menée par la France au cours de notre siècle a précisément concerné l'Alsace- Lorraine.

6 C'était là le temps où les nations se barricadaient chez elles, toujours promptes à voir dans le voisin un féroce soldat en puissance, prêt à venir égorger les fils et les compagnes de leurs propres soldats. On pourrait croire qu'aujourd'hui les attitudes sont plus détendues et permettent enfin de commencer à exploiter les possibilités de contacts inhérentes à ces héritages linguistiques qu'il n'est plus nécessaire de suspecter. Que l'on peut, par exemple, valoriser en Alsace ou en Catalogne Nord, l'usage de langues 
«locales» ouvrant sur des espaces voisins, partenaires en puissance : compte tenu du nombre de travailleurs qui franchissent la frontière chaque jour en Alsace ou compte tenu de la puissance d'attraction de la métropole barcelonaise, on voit qu'il n'est pas ici question seulement de culture ou d'identité, mais bel et bien d'armer les populations locales face aux demandes induites par leur insertion dans un cadre socio-économique transnational. Dans un autre domaine, faudra-t-il attendre la mort de l'occitan pour comprendre enfin ce qu'écrivait Jean Jaurès, en 1911, sur la parenté entre la langue d'oc et les langues romanes d'Europe du sud, et les possibilités d'apprentissage de ces langues qu'ouvrait aux enfants du Midi cette parenté ? Ainsi, même en fonction des critères utilitaires qui régissent aujourd'hui la question de l'apprentissage des langues, on saisit à quel point les « vieux patois » peuvent avoir encore leur mot à dire. Mais tout le monde le saisit-il vraiment?

\section{La charte européenne des langues et cultures régionales}

7 Au niveau européen, on assiste depuis quelques années à d'indiscutables progrès dans la prise en compte des réalités linguistiques des États de la Communauté. Ce n'était certes pas l'urgence dans les premiers temps de la construction européenne, mais le problème a fini par émerger. Le Parlement européen, à partir de propositions déposées dès 1979 par des députés, relayées en 1981 par un rapport dû au député italien Arfè, a adopté le 16 octobre 1981 une résolution demandant aux États membres de mettre en place une politique de soutien aux langues « régionales » (c'est le terme employé) dans le domaine de l'enseignement, des médias et de la vie publique, et appelant à la rédaction d'une « charte communautaire des langues et cultures régionales ». L'année suivante, est créé un Bureau européen des langues moins répandues, réunissant des experts issus des diverses minorités européennes, financé par la Communauté et travaillant en liaison avec ses diverses instances - Commission et Conseil des ministres. L'action ainsi entreprise s'est poursuivie depuis, avec des hauts et des bas. Il est vite apparu que tous les États membres n'étaient pas disposés à s'engager dans la direction préconisée par la résolution de 1981. Du coup, le Parlement européen s'est vu saisi à intervalles réguliers de propositions de résolution destinées soit à attirer l'attention sur des situations particulières - Catalogne, française ou espagnole, Frise néerlandaise... - soit à relancer l'idée d'une charte européenne manifestement ignorée par les instances exécutives. Et c'est dans une autre instance européenne, plus large, mais aussi moins directement liée par nature à la dynamique de la construction de la Communauté des Douze, le Conseil de l'Europe, qu'ont été finalement accomplis les efforts les plus grands. Le Conseil avait lancé dès 1978, dans la déclaration de Bordeaux, l'idée d'une charte des droits culturels des minorités en Europe et c'est finalement grâce à lui que cette charte a pu progressivement être rédigée, puis adoptée successivement, à partir de 1988, par la Conférence permanente des pouvoirs locaux et régionaux, puis par l'Assemblée parlementaire, avant d'être enfin adoptée dans sa forme définitive par le Comité des ministres du Conseil de l'Europe le 22 juin 1992, et soumise à la ratification définitive des États membres.

8 La lecture de cette Charte, dans ses versions successives ${ }^{1}$ révèle bien la difficulté pour les instances européennes de produire un texte acceptable par tous les gouvernements. La Charte entend définir la place des langues « régionales ou minoritaires » dans un certain nombre de domaines: enseignement (partie III, article 8), justice (article 9), services 
publics (article 10), médias (article 11), activités et équipements culturels - bibliothèques, musées, archives, théâtre, production littéraire et cinématographique, etc. - (article 12), vie économique et sociale (article 13). Dans chaque domaine, la Charte fait des propositions - mais des propositions modulées, définissant des degrés dans la prise en compte des langues minoritaires, et laissant à chaque État la liberté de choisir jusqu'à quel degré il ira dans cette prise en compte. Ainsi dans le domaine de l'enseignement, un État pourra, soit "prévoir un enseignement (primaire, secondaire, technique ou supérieur) assuré dans les langues régionales ou minoritaires concernées" (version 1992; la version 1991 précisait "assuré totalement»); soit "prévoir qu'une partie substantielle de l'enseignement [...] soit assurée dans les langues [...] concernées »; soit intégrer ces langues au curriculum scolaire; soit enfin « appliquer l'une des mesures visées ci-dessus au moins aux élèves dont les familles le souhaitent et dont le nombre est jugé suffisant ». Comme on voit, ce qui est ici proposé, c'est une politique «à la carte », où chaque État peut choisir l'article qui convient le mieux à son métabolisme culturel particulier.

9 Le même scénario - ou plutôt le même éventail de scénarios possibles - se retrouve dans chacun des autres articles. On pourra ainsi accepter que toutes les procédures de justice soient menées dans la langue minoritaire, ou que toutes les autorités administratives utilisent cette même langue, ou qu'elle soit dotée d'une chaîne de télévision, à moins que l'on ne préfère se contenter « d'établir dans ces langues régionales ou minoritaires, sur demande, les actes liés à une procédure judiciaire ", ou d'autoriser les locuteurs des dites langues à soumettre à une administration un document rédigé en leur langue, ou de veiller à ce que les radios et télévisions veuillent bien programmer des émissions en langue régionale. Et tout à l'avenant. D'une manière générale, on se trouve devant une fourchette largement ouverte, entre le scénario optimal (la langue minoritaire est acceptée à part entière) et un scénario beaucoup plus modeste (la langue « régionale » est tolérée, à condition de demeurer courtoisement dans les marges du système - et le citoyen peut l'utiliser, à condition d'en manifester explicitement le désir : rien ne lui est accordé a priori). Et en tout état de cause, c'est l'État national et lui seul qui décide.

Il n'y a là rien de bien contraignant, on en conviendra. Pourtant, la rédaction de cette Charte a été extrêmement difficile et il a fallu plus de dix ans pour en venir à bout. C'est que les États - un certain nombre d'entre eux du moins - ont manifesté un très grand manque d'enthousiasme. La Grèce, la Turquie et... la France, entre autres, ont rogné efficacement les aspérités initiales du texte. Nous ignorons bien sûr les marchandages qui ont pu accompagner, en haut lieu, ses énoncés originels. Nous ne pouvons juger qu'à partir de ce qui a été publié pour la version de 1991 et pour la version finale. Mais cela suffit pour relever un certain nombre d'interventions non dépourvues de portée. Un article 8 prévoyait initialement que les Parties s'engageaient à « éliminer [...] toute forme de discrimination en ce qui concerne l'utilisation des langues et cultures régionales». Dans la version finale soumise à la ratification des divers États, cet article a disparu en tant que tel et n'apparaît plus que comme paragraphe inclus dans l'article 7 ; au surplus, l'article 21 stipule que tout État pourra formuler une ou plusieurs réserves concernant ce paragraphe, au moment de la ratification : autant dire que la disposition se retrouve ainsi largement dépourvue de sa signification initiale. 


\section{Une politique linguistique française hésitante}

11 Mais il faut croire que, même patiemment amendée par d'ingénieux diplomates, cette Charte continue à sentir le soufre. Car si le Comité des ministres a fini par l'adopter, tous les États ne l'ont pas ratifiée, lui interdisant de fait d'entrer en vigueur sur leur sol. Parmi ces réfractaires, on retrouve la Grèce, la Turquie... et la France. Malgré d'occasionnelles promesses ministérielles, avant comme après le changement de majorité de mars 1993, la France n'a pas voulu adopter la Charte. Cette attitude peut étonner. Grecs et Turcs sont en l'occurrence fidèle à une longue tradition de négation des problèmes minoritaires sur leur sol - la Grèce, pour ne parler que d'elle, montre autant d'enthousiasme à dénoncer l'oppression des Grecs d'Albanie qu'à rejeter toute question embarrassante sur les Albanais de Grèce. Mais la position de la France est plus inattendue. Sa réticence à l'égard du problème des minorités est évidente et ancienne, mais ne prend pas la forme parfois excessive qu'elle peut revêtir à Athènes ou à Ankara. Mieux : au fond, les dispositions minimales proposées par la Charte en matière d'enseignement ou de médias sont déjà appliquées en France depuis assez longtemps, fût-ce avec une mauvaise volonté remarquable. Rien en apparence dans les propositions de la Charte n'aurait dû choquer le gouvernement français. Alors?

Alors, il nous parait que deux choses peuvent expliquer ce refus têtu. En premier lieu, les dispositions pratiques du document s'agrémentent d'un complément, en forme d'instance de contrôle. Il est prévu, dans la partie IV de la Charte, que chaque État doit présenter périodiquement au secrétaire général du Conseil de l'Europe un rapport sur la politique qu'il a suivie vis-à-vis de ses minorités. Ce rapport sera soumis à un comité international d'experts et il sera possible à des associations minoritaires d'y joindre leurs remarques et doléances éventuelles. Le tout, transmis au Comité des ministres, pourra donner lieu, de la part de ce dernier, à des « recommandations » à l'adresse de tel ou tel État membre. Dès lors, on commence à comprendre les réticences de la France, qui considérerait de telles « recommandations » comme une désagréable ingérence dans ses affaires intérieures. Et qui considère apparemment, a priori, que le contenu de sa politique à l'égard des cultures minoritaires de son territoire n'a pas à donner prise à des critiques de la part d'autres pays.

On touche ici au deuxième élément d'explication du refus français. Pour l'idéologie nationale, il n'existe pas de «minorités » en France, puisque tous les citoyens sont égaux. Définir telle ou telle fraction de la population comme une minorité, ou comme "un peuple» (qu'on se souvienne des débats autour du "peuple corse »), c'est d'emblée mettre cette fraction hors du lot commun, donc porté atteinte au principe d'égalité. Cette attitude traditionnelle explique le vote tout récent d'un député français élu au Parlement européen, le professeur Schwarzenberg, qui a été le seul à voter contre l'adoption par le Parlement, en février 1994, du rapport Killilea concernant la politique européenne à mettre en œuvre en matière de minorités - un rapport de plus. Le professeur Schwarzenberg a justifié son vote par une distinction soigneuse entre deux niveaux : celui des traditions familiales - autant dire du domaine privé - où la survivance des langues régionales ne le gêne nullement. Et le niveau national, où ce qui doit l'emporter, c'est la défense de l'unité française. Une unité que les divers textes européens mettent apparemment en danger, si on l'en croit. Cette prise de position, toute isolée qu'elle paraisse, nous semble assez bien correspondre, en profondeur, à l'état d'esprit de 
beaucoup d'hommes politiques français - de tous bords - sur la question, même si peu affichent aussi nettement leur opinion. Au fil des ans, certes, les autorités ont pu faire, sans réel enthousiasme, quelques concessions sur l'enseignement ou les médias. Mais toujours au coup par coup et dans des limites relativement étroites. Et surtout par voie réglementaire plus que législative. Car une loi risquerait de faire apparaître au grand jour, et de soumettre à débat, un fait minoritaire que l'on entend minimiser au maximum. On préfère donc cette manifestation du fait du prince qu'est la circulaire ministérielle à l'expression d'une volonté des élus du peuple souverain. La Charte européenne mettant Corses, Occitans, Bretons et autres au rang des "minorités " reconnues ailleurs Catalans, Gallois, sud-Tyroliens, etc. - et les plaçant sous la garantie d'un texte international, l'adopter serait rompre de fait avec l'attitude jusqu'ici suivie et qui ne satisfait manifestement pas les principaux intéressés ; refuser de ratifier, c'est se donner la possibilité de persister dans le traitement traditionnel de la question des «cultures régionales ", chez soi, sans regards indiscrets.

fond de tout ceci, on retrouve ce qui constitue une des contradictions principales de l'attitude actuelle de la France vis-à-vis de l'Europe: l'hésitation constante entre deux attitudes. D'un côté, le désir de s'intégrer à un espace plus vaste, susceptible de protéger le pays contre les aléas de la compétition mondiale - alors même que réduit à ses seules forces il risque de ne plus faire le poids. Et d'un autre côté, le poids de l'histoire: la France, après tout, n'est-elle pas le modèle des États-nations ? N'importe-t-il pas de tenter de préserver ce qui fait sa singularité ? On n'a pas ici seulement affaire à l'opposition banale des « pro-européens » et des « eurosceptiques » pour le dire à l'anglaise : c'est au cœur même des secteurs les plus acquis à l'idée européenne qu'on peut voir apparaître, à certains moments, le signe de la contradiction.

Par exemple, à travers tout ce qui peut concerner la politique linguistique. On l'a vu avec la révision de la Constitution intervenue en 1992 en prélude à la ratification du traité de Maastricht, quand la table de la Loi s'est vue enrichie, dans son article 2, de l'affirmation solennelle $\mathrm{du}$ fait que la langue nationale est le français - ce qu'aucun texte constitutionnel n'avait jusqu'ici jugé bon de préciser, tant cela semblait l'évidence. Pour une France intégrée à une Europe nouvelle, qu'elle est bien incapable de dominer économiquement ou politiquement - glissons sur la dimension militaire... -, la question de la langue commence bel et bien à se poser. D'où l'actuel projet de loi sur l'emploi de la langue française, justifié en ces termes par le ministre de la culture :

La richesse de toutes les langues doit être mise en valeur. Le pluralisme linguistique doit être préservé et organisé. Entre pays européens de langues différentes, il faut savoir résister aux séductions des arguments en faveur d'une langue véhiculaire unique qui entraînerait, à terme, le ravalement de toutes les langues sauf une au rang de langue locale. (Le Monde, 24 février 1994)

Nobles propos, auxquels tout défenseur d'une "petite langue " pourrait sans embarras souscrire. Mais il n'est pas question, dans l'esprit de leur auteur, des "petites langues ». Quand M. Toubon parle de plurilinguisme, il revendique une place pour le français aux côtés de l'anglais triomphant. Et son projet de loi a surtout pour fonction d'ériger un mur de tabous lexicologiques autour d'une langue française décrite comme en péril. Nous n'avons pas à juger ici de la pertinence de ce texte, et nous laissons à ses partisans la tâche de vérifier par eux-mêmes si la répression suffit à contrôler les flux du marché linguistique. Bornons-nous à noter que, malgré quelques bonnes paroles, la sauvegarde du français n'est nullement associée à celles des langues régionales. La langue nationale est première servie. 
17 Tout se passe donc comme si, avant de se laisser embarquer dans l'Europe, la France entendait bien se munir préventivement et à tout hasard de ses propres chaloupes de sauvetage. Et comme si le dépassement de la nation constituait un déchirement : ce qu'il est sans nul doute, tant l'idéologie nationale fait partie du paysage mental du pays. Du coup, on conçoit que la question des langues régionales ou minoritaires pèse peu : c'est au mieux un problème annexe, sans commune mesure avec le drame qui frappe l'héritage hexagonal dans son entier. Car on ne saurait prétendre, n'est-ce pas? Que les menaces qui pèsent sur le breton ou le basque sont plus graves et moralement plus condamnables que celles qui pèsent sur la pureté du vocabulaire! Et s'il est scandaleux qu'un jeune lycéen de Quimper dise walkman ou has-been au lieu de dire Dieu sait quoi (nous renvoyons le lecteur puriste au Journal officiel adéquat), il est apparemment tout à fait normal qu'il ne comprenne plus les noms de lieux qui entourent sa ville, pour cause d'ignorance du breton. Au pire, les langues «régionales » pourront même être perçues comme ayant partie liée avec l'avancée sournoise des idiomes concurrents. Le ministre de l'éducation nationale, après avoir prononcé devant le Conseil général de son département un fort beau discours, en occitan, à la gloire des langues de France, s'est jusqu'ici bien gardé de déposer le moindre projet de loi en leur faveur. Il a ainsi retrouvé comme par hasard les méthodes de ses prédécesseurs, lointains ou immédiats, qui n'abordaient le sujet qu'en termes choisis, et jamais de façon à se trouver ensuite trop liés. Gageons que pour les uns comme pour les autres, c'était le moyen d'éviter de trop vives polémiques².

En résumé, l'ouverture sur l'Europe, jusqu'ici, a pu jouer un rôle au niveau d'une certaine sensibilisation internationale au problème des langues moins répandues, mais, en ce qui concerne la France en tout cas, elle n'a pu contrebalancer l'effet d'une tradition séculaire de refus de poser le problème.

Toutefois, on ne saurait réduire le débat à la seule dimension d'une contradiction entre une Europe humaniste, pleine de sollicitude à l'égard des pauvres petites langues moribondes du continent, et des États-nations endurcis dans le péché, repliés hargneusement sur leur nationalisme linguistique, toujours prêts à enfoncer les " patois » qui ont le mauvais goût de survivre sur leur sol sacré. L'action du Conseil de l'Europe est restée jusqu'ici purement théorique et c'est surtout à la Communauté qu'il appartiendra de prendre des mesures plus concrètes. Et, de toute façon, toutes les chartes du monde, nanties de toutes les signatures possibles, promises à l'application la plus scrupuleuse, ne suffiront pas à sauver les langues régionales et minoritaires, en France et ailleurs, si un certain nombre de facteurs ne viennent pas en renfort.

\section{Faire vivre les langues de France}

Premier élément: jusqu'à quel point peut-on sauver certaines de ces langues? Leurs défenseurs, dont nous sommes, on l'aura compris, n'aiment pas se poser cette question. Ce n'est pas une raison pour l'esquiver. L'éventail des mesures proposées par la Charte n'est pas seulement le résultat d'âpres marchandages. Il est aussi la prise en compte pragmatique de la diversité des situations réelles et des possibilités concrètes qui s'offrent à chaque langue donnée. Dans le cas des langues de France, on imagine mal qu'on puisse exiger d'un tribunal qu'il utilise dans sa procédure l'occitan ou le breton: l'état de la pratique de ces derniers, mais aussi le traditionnel partage des rôles entre langues, accepté depuis si longtemps par les juges comme par les justiciables, font que la société dans sa masse n'entend pas rompre avec les habitudes maintenant acquises. Tout 
au plus est-il légitime et possible de revendiquer le droit pour l'accusé à «s'exprimer dans sa langue régionale ou minoritaire ", même s'il connaît par ailleurs parfaitement le français : ce qui permettrait aux cours d'assises d'éviter les débats qui ouvrent souvent les procès de militants basques ou corses. En tout état de cause, il est de peu de profit de méconnaître l'état réel des langues concernées, et de vouloir « faire comme si » elles se portaient mieux que ce n'est le cas. Il n'est donc de politique possible que réaliste, s'attachant à développer les espaces de parole accessibles aux langues de France, du fait de leur situation sociolinguistique actuelle, quitte à franchir de nouvelles étapes s'il se révèle que, cette situation s'améliorant, de telles avancées deviennent possibles.

21 À l'heure actuelle, les premières enquêtes sur l'état de ces langues et, surtout, sur l'image que s'en font leurs locuteurs, commencent à être analysées. Elles révèlent un paysage très contrasté. Le déclin, comme langue d'usage social large, de l'occitan, du breton, voire même de l'alsacien, longtemps préservé, se voit confirmé. Le breton, en 1991, est parlé par $21 \%$ de la population de la Bretagne bretonnante. En Languedoc-Roussillon, l'occitan est parlé par un pourcentage analogue des habitants d'une région qui, à vrai dire, a vu son peuplement bien bouleversé depuis quelques décennies. Un locuteur sur quatre ou sur cinq, ce n'est pas énorme, même en tenant compte du fait que, dans les deux cas cités, pas loin de la moitié de la population semble apte à « comprendre » la langue régionale. C'est d'autant moins énorme que ce dont les chiffres bruts ne rendent pas compte, c'est de la réalité des pratiques, à distinguer de celle des compétences. En d'autres termes, savoir parler breton ou occitan n'implique pas qu'on le parle quotidiennement, ni dans toutes les occasions, ni à tout le monde. Quant au degré de transmission de la langue des parents aux enfants, toutes les enquêtes concordent pour le placer assez bas, voire pour l'estimer à zéro : dans le village bigouden de Plozevet, rendu illustre il y a bien des années par une enquête « anthropologique » qui avait vaillamment ignoré le problème de la langue, une étude de 1993 permet de voir que si le breton est la langue maternelle de $100 \%$ des plus de 50 ans, ce pourcentage tombe à $0 \%$ des moins de 25 ans. On mesure ici à quel point les dernières décennies ont été dramatiques pour les langues minoritaires de France ${ }^{3}$.

Faut-il alors en conclure qu'il est trop tard, et que la question est réglée? Le second enseignement des enquêtes bretonne et occitane que nous venons d'évoquer, c'est l'amélioration de l'image des deux langues concernées. $92 \%$ des sondés de l'enquête TMO de 1991 pensent qu'il faut conserver le breton. $82 \%$ pensent qu'il doit avoir sa place à l'école - ce qui n'empêche d'ailleurs nullement les mêmes de se montrer peu optimistes quant aux possibilités réelles de sauver la langue. Les chiffres du Languedoc-Roussillon dessinent les mêmes tendances de fond. Les parents et les grands-parents des personnes sondées avaient fait de grands efforts pour assurer à leur progéniture la maîtrise du français, passeport de la promotion sociale, quitte à délaisser le « patois » au passage. Au bout de ce processus, on se trouve face à des gens qui le déplorent et manifestent avec plus ou moins de conviction le souhait de le voir s'inverser. Ce paradoxe montre à tout le moins que le sort des langues minoritaires n'est pas du tout réglé de façon définitive. Et il serait intéressant d'avoir une enquête de même type pour le corse, dont la situation est tout à fait particulière.

23 C'est dans cet état que les langues de France se préparent à devenir langues d'Europe : elles ne sont pas vraiment en bonne santé et leur place dans les pratiques langagières réelles, dans la vie sociale, est assez restreinte. Mais elles survivent, non seulement dans leurs derniers réduits d'usage spontané, mais aussi dans une bonne partie des sociétés locales, comme souvenir, ou comme objet de désir, ou comme emblème de l'identité 
régionale. Sans oublier le fait qu'elles demeurent des outils de création pour des écrivains ou des chanteurs, et qu'elles entrent dans le bagage scolaire d'un nombre non négligeable d'enfants, malgré les difficultés liées au caractère longtemps facultatif de leur enseignement et à la concurrence que de récentes réformes instituent entre elles et les langues vivantes étrangères. Au bout du compte, on aurait tort de comparer leur état à celui de langues minoritaires mais reconnues comme le gallois ou le catalan; mais on aurait tort, symétriquement, de les enterrer sans phrases.

Quelle place pour elles, alors, dans l'Europe de demain? Au pire, celle de vestiges vénérables que l'on visitera avec une ombre de nostalgie, comme les Gaeltachtai d'Irlande, ces réserves linguistiques situées dans les zones occidentales les plus déshéritées de la République, peuplées de gens qui bien souvent ne rêvent que de les quitter, et que l'Irlandais moyen, le plus souvent anglophone monolingue, salue distraitement comme les réceptacles pittoresques de l'identité ancestrale. Mais le pire est-il toujours sûr? Et ne peut-on imaginer pour ces langues un destin moins funèbre? Nous l'avons dit, mais il faut le rappeler : dans bien des cas, les langues de France ne sont pas des culs-de-sac n'ayant d'autres débouchés qu'elles-mêmes. Elles sont liées à des langues voisines, parfois prestigieuses. La fin de la méfiance entre les vieux États-nations peut à présent les revivifier. La germanophonie de l'Alsace et de la Lorraine thioise cessera alors d'être objet de méfiance policière et patriotique pour devenir atout dans l'intégration de la France de l'est à l'arc rhénan. Et à l'autre bout de l'hexagone, on pourra voir la fin de l'actuel paradoxe d'un catalan officiel au sud, s'étalant sur tous les panneaux routiers ou publicitaires du Principat, flanqué au nord d'un catalan clandestin, ravalé au rang de " patois roussillonnais » par de bons esprits français qui en ignorent tout, au demeurant.

Mais au-delà, il y a encore plus important peut-être que ce point de vue somme toute purement utilitaire. La construction d'une véritable communauté européenne, enracinée dans autre chose que de laborieux accords commerciaux et monétaires, sera ardue. La diversité des traditions politiques, des histoires, des cultures et des mentalités, s'ajoutant à la disparité des forces économiques : autant de facteurs susceptibles de la ralentir. Il faudra faire des concessions, et admettre l'autre, dans sa différence autant que dans sa similitude. Si la France doit conserver, à l'égard des autres langues du continent, l'attitude d'ignorance méprisante qu'elle a appliquée aux langues de son sol depuis le XVI ${ }^{e}$ siècle au moins, mieux vaut arrêter les frais tout de suite - ne serait-ce que parce que si l'État monarchique, puis républicain avait les moyens d'imposer sa langue à ses sujets, la place de la France dans le monde n'est plus si importante qu'elle puisse lui permettre d'appliquer la même politique à l'allemand ou à l'italien - et on ne parle même pas de l'anglais. Il va falloir guérir de cette vieille maladie si agréable, le francocentrisme naif. Dans cette cure inévitable et souhaitable, le traitement des langues de France pourra constituer un bon entraînement.

Un dernier point. La balle est sans doute dans le camp du français; mais les défenseurs des langues de France auraient tort de croire qu'ils n'ont rien d'autre à faire qu'à attendre - et à solliciter, avec l'insistance dont ils ont l'habitude - un changement d'attitude de la part du pouvoir central. Une langue, une culture ont certes, dans l'abstrait des grands principes, droit à la vie. Mais cela implique aussi d'être capable de vivre, et de vivre l'échange créatif avec les autres cultures. Les fenêtres de la Maison France ne sont pas seules à devoir s'ouvrir au vent d'Europe. Si les Bretons, les Corses, les Basques, les Occitans n'ont rien d'autre à proposer que la célébration close de leur identité et la déploration geignarde de la situation dans laquelle on l'a mise, leur avenir est tout tracé : 
c'est celui du gaeltacht que nous évoquions plus haut. L'action du Bureau européen pour les langues moins répandues a déjà permis à des Occitans ou à des Bretons de voyager à travers l'Europe pour aller voir ailleurs comment d'autres concevaient et réalisaient des politiques scolaires ou culturelles au service de leurs langues. Voilà un moyen efficace d'élargir les horizons. Et c'est au prix de cet élargissement que les langues de France, comme les autres petites langues d'Europe, peuvent avoir une chance de se faire entendre dans le concert des cultures européennes qui prélude.

\section{NOTES}

1. Nous utilisons ici le texte de la Charte tel qu'il est donné, dans sa version de juin 1991, par l'ouvrage collectif Les Minorités en Europe, Henri Giordan (dir.), Paris, Kimè, 1993, p. 585 sqq., et dans sa version définitive (juin 1992) qui nous a été communiquée à l'époque par l'antenne française du Bureau européen pour les langues moins répandues.

2. À qui douterait que le sujet puisse vraiment susciter de vives réactions, on ne peut que signaler les cris d'orfraie qui avaient salué, en 1982, la sortie du rapport Giordan dont l'objet était précisément de définir la place des «langues régionales dans la culture nationale » et de préconiser les mesures législatives à prendre en leur faveur.

3. Cf. Francis Favereau, Bretagne contemporaine, Skol Vreizh, 1993, p. 28 sqq. L'enquête réalisée en Languedoc-Roussillon en 1991, par sondage, révèle une situation similaire en ce qui concerne les générations les plus jeunes. En Alsace, une enquête de 1986 faisait apparaître à la fois une certaine résistance de l'alsacien comme langue maternelle - $9 \%$ des adolescents d'alors n'avaient pas connu d'autre langue avant d'entrer à l'école - et une détérioration rapide de ses positions ( Le déclin du dialecte alsacien, M.N. Denis, C. Veltman, Strasbourg, 1989).

\section{RÉSUMÉS}

Comment la politique linguistique française à l'égard des langues régionales se situe-t-elle par rapport à la politique du Conseil de l'Europe dans ce domaine ? La question est examinée à partir du postulat qui rappelle le caractère transfrontalier - et, donc, européen - des langues régionales parlées en France.

\section{INDEX}

Index géographique : France, Europe

Mots-clés : charte européenne des langues régionales ou minoritaires 


\section{AUTEUR}

\section{PHILIPPE MARTEL}

CNRS, université Paul Valéry de Montpellier 